\title{
Formulation and In-Vitro evaluation of Nanocrystal formulation of poorly soluble drugs
}

\author{
Arvind Sharma 1*, Alok Pal Jain ${ }^{2}$, Sandeep Arora ${ }^{3}$ \\ ${ }^{1}$ Associate Professor, Shiva institute of B. Pharmacy, Bilaspur Himachal Pradesh, India \\ 2 Principal RKDF Collge of Pharmacy, SRK University, Bhopal, India \\ ${ }^{3}$ Director Chitkara College of Pharmacy, Chitkara University, Punjab, India
}

\begin{abstract}
Introduction:-Poor solubility of drug compounds which accounts for $40 \%$ of new molecules investigated at present is an issue of great concern in pharmaceutical industry and reducing particle size (i,e to reduce below $1000 \mathrm{~nm}$ )of drug candidate to be investigated is one of the simplest and efficient ways to overcome this challenge. Drug nanocrystals, solid nanosized drug particles are defined as formulation having $100 \%$ drug, which are covered by a stabilizer layer. In this study attempt was made to formulate and evaluate nanocrystals of poorly soluble drugs having low oral bioavailability. Material and method:- Nanocrystals were prepared successfully by varying concentration of different stabilizers by anti-solvent precipitation method. The formulated nanocrystals were evaluated by determining physicochemical characteristics such as physical appearance, Differential Scanning Calorimetry (DSC), scanning electron microscopy (SEM), X-ray powder diffractometry, solubility studies, particle size distribution, zeta potential, and in vitro drug release profile studies. Results:- An in-vitro study was performed on the successful formulation in comparison to drug powder using dissolution apparatus The particle size of RVT and PSNC-3 was found to be 1975.3 $\mathrm{nm}$ and $790.1 \mathrm{~nm}$ respectively. Conclusion: Precipitated Nanocrystals formulated with different stablizer's method resultedin formation of small and uniform RVT nanocrystals with an improved saturation solubility, dissolution rate.
\end{abstract}

Keywords: Nanocrystal, poorly soluble drugs

Article Info: Received 29 June 2019; $\quad$ Review Completed 11 Aug 2019; $\quad$ Accepted 19 Aug $2019 ; \quad$ Available online 25 August 2019

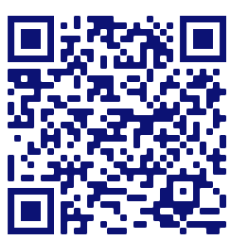

Cite this article as:

Sharma A, Jain AP, Arora S, Formulation and In-Vitro evaluation of Nanocrystal formulation of poorly soluble drugs, Journal of Drug Delivery and Therapeutics. 2019; 9(4-s):1183-1190～DOI: http://dx.doi.org/10.22270/jddt.v9i4-s.3873

*Address for Correspondence:

Arvind Sharma, Associate Professor, Shiva institute of B. Pharmacy, Bilaspur Himachal Pradesh, India

\subsection{INTRODUCTION}

Currently, poor solubility which is presently estimated for $40 \%$ of new molecules investigated is an issue of great concern in drug discovery and formulation development, to combat the issue of poor aqueous solubility of hydrophilic drugs many techniques are explored viz at molecular level prodrugs ${ }^{[1]}$, salt formation [2], co-solvent systems ${ }^{[3]}$ or cyclodextrins [4] ,co-crystals [5], amorphous systems [6] or particle size reduction [7] have been studied widely. A another approach which can be explored for enhancement of solubility as well as dissolution are documented as colloidal systems, like SEDDS/SMEDDS/SNEDDS[8] (micro/nano)emulsions [9] or they may be different kinds of other lipid based systems like vesicular systems[10]. Based on the physicochemical characterization properties of the drug candidate, the most appropriate way to improve and enhance the solubility by above stated may be selected $[11,12,13]$ Efficient screening methods explore number of poorly soluble drug molecules for pharmaceutical industry to commercialize, as it has been well documented that approximated ninety percent of the new chemical entities are having poor aqueous solubility. According to Biopharmaceutics Classification System (BCS), these drug candidates or compounds are categorized as class II $(70 \%)$ or class IV $(20 \%)[14,15]$

As classified in BCS system, drugs belonging to class II are well permeable but poorly soluble, therefore are excellent \& potential candidates to be formulated as drug nanocrystals (NC )[16,17], poorly soluble and poorly permeable drugs which are categorized as BCS class IV as documented may be benefited from decreased size [18,19]. For example, higher dissolution velocity resulted from higher and faster drug solubility a gradient on higher concentration side i develop between the intestine and lumen which consequently improved drug permeation and in turn improved bioavailability. Drug nanocrystals are drug 
particles in nano range (i,e below $1000 \mathrm{~nm}$ ), which are have sheath of stabilizer layer, are mostly exploited for enhancing the properties such as solubility of poorly soluble drugs [20]

The main aim of current study is to enhance the dissolution rate, solubility as well as bioavailability of drug of the Rosuvastatin (RVT), a poorly aqueous soluble drug by preparing nanocrystals using antisolvent precipitation technique. RVT crystalline nature can be considered as factor for reduction in aqueous solubility as well important parameter in reduction of oral bioavailability which is reported to be $\leq 20 \%$. Rosuvastatin when administered orally, $\mathrm{C}_{\max }$ (peak plasma Concentration) is reached within 3-5 $\mathrm{h}, \mathrm{V}_{\mathrm{d}}$ (volume of distribution ) is $1.1-1.4$ liter $/ \mathrm{Kg}$, and plasma protein binding is reported to be $90 \%$. [21].

\subsection{MATERIAL AND METHODS}

2.1 Materials. Rosuvastatin calcium was gifted by Microlabs Pvt. Ltd.,Ethanol , Ploxomer-88, sodium Lauryl sulphate (SLS)were purchased from S.D. Fine Chemicals, Mumbai, India., , Span 80, Tween 80, Acetonitrile and Methanol were purchased from Sigma Aldrich, USA.

\section{Methods 2.2}

\subsubsection{Ultraviolet Absorption Maxima ( $\left.\lambda_{\max }\right)$}

The $0.01 \% \mathrm{w} / \mathrm{v}$ solution $(100 \mu \mathrm{g} / \mathrm{ml})$ of the Rosuvastatin Calcium in PBS (6.8), methanol, acetonitrile was scanned between 200-400nm and absorption maximum was determined spectrophotometrically (Systronic 2202, Ahmedabad, India).

\subsubsection{Solubility Studies}

Saturation solubility of pure drug Rosuvastatin as well as precipitated nanocrystals was estimated by method reported by Higuchi. Each sample in access amount was added to ten $\mathrm{ml}$ of different media $(0.1 \mathrm{~N} \mathrm{HCl})$, Phosphate Buffer (PBS) pH (6.8 and pH 7.4) in glass vials. These vials were then kept in orbital shaker for $48 \mathrm{~h}$ at $37^{\circ} \mathrm{C}$. The solution was then filtered using syringe filter $(0.22 \mu \mathrm{m})$, and the absorbance was taken using an ultraviolet (UV) spectrophotometer to determine the amount of drug dissolved..

\subsubsection{Formulation of nanocrystals}

Nanocrystals of RVT were prepared successfully using different polymeric stabilizers viz. Sodium Lauryl Sulphate, polaxmer 188 etc ,y antisolvent precipitation method. 100 mg of pure drug was dissolved in $10 \mathrm{ml}$ of Acetonitrile (ACN) (Solution I). Stabilizing agents were dissolved in $100 \mathrm{ml}$ of double distilled water (Solution II). This Solution II was placed under propeller mixer at constant speed of $1400 \mathrm{rpm}$. Then, drug solution (Solution I) was injected into Solution II which is placed on magnetic stirrer with continuous stirring at $1000 \mathrm{rpm}$. Rotation per minute 500 was maintained overnight for complete removal of solvent. Once the organic solvent is removed the solution was then centrifuged at $5000 \mathrm{rpm}$ and supernatant solution so obtained was subjected to solidification by lyophilization by which water under vaccum is removed from precipitated nanocrystals when it is in frozen state (Allide Frost,New Delhi,India) at $40^{\circ} \mathrm{C}$ for 24 hours to obtain the drug nanocrystals.
Table 1: Formulations with different ratios of drug: stabilizer

\begin{tabular}{|l|l|l|}
\hline $\begin{array}{l}\text { Formulation } \\
\text { code }\end{array}$ & Stabilizer & $\begin{array}{l}\text { Drug:Stabilizer } \\
\text { ratio }\end{array}$ \\
\hline PNC-1 & Polaxmer 188 & $1: 1$ \\
\hline PNC-2 & & $1: 2$ \\
\hline PNC-3 & & $1: 3$ \\
\hline SNC-1 & SLS & $1: 1$ \\
\hline SNC-2 & & $1: 2$ \\
\hline SNC-3 & & $1: 3$ \\
\hline PSNC-1 & SLS: Polaxmer 188 & $1: 1$ \\
\hline PSNC-2 & & $1: 2$ \\
\hline PSNC-3 & & $1: 3$ \\
\hline
\end{tabular}

PNC= Polaxmer nanocrystals, $\mathrm{SNC}=$ Sodium lauryl sulphate nanocrystals, $\mathrm{PSNC}=$ Polaxmer Sodium lauryl sulphate nanocrystals

\subsubsection{Estimation of Particle Size Distribution and Zeta Potential (ZP)}

Zetasizer (Nano ZSTM Malvern Instruments, UK) was used for analysis of particle size and their distribution pattern of drug nanocrystals using a process called dynamic light scattering (DLS). Parameters such as mean particle size, size distribution, and polydispersity index (PDI) were examined and determined. The particle size distribution (PSD) was characterized using PDI, which is a measure of the width of size distribution. Measurement of zeta potential of samples was performed by Zetasizer (Nano ZS TM Malvern Instruments, UK) using standard conductivity to avoid variation due to conductivity of $\mathrm{H}_{2} \mathrm{O}$ because in some instance drug candidate may contain electrolytes. The nanosuspension samples were measured in standard conductivity which was adjusted 1:1 (Distilled water) as well as original media in order to measure thickness of diffusion layer at $25^{\circ} \mathrm{C}$ for zeta potential and results were performed in triplicate.

\subsubsection{Crystalline state evaluation:}

Powder X-Ray diffraction (PXRD) and Differential Scanning Colorimeter (DSC) techniques were used to evaluate the crystalline nature of Drug Nanocrystal formulations [22].

\subsubsection{Differential Scanning Calorimetry (DSC):}

Differential Scanning Calorimeter Perkin-Elmer DSC-7 was used to evaluate thermal properties of the pure drug as well as precipitated drug nanocrystals. It is well documented that amount of sample to be investigated and analyzed shall range between 4 to $7 \mathrm{mg}$ and should be placed in crimped aluminum sealed 50 microliter pans. Each sample subjected to analysis was exposed to heat runs which were set between 50 to 300 degree Celsius maintained at rate of scanning $10^{\circ} \mathrm{min}^{-1}$ so that quick redraw and image is available after display, under dry nitrogen flow (100 $\mathrm{ml} / \mathrm{min})$.

\subsubsection{Powder X-ray diffraction (PXRD):}

Diffractograms of every formulation and pure drug were observed recorded by technique PXRDusing (Philips analytical XRD PW3710) with radiation source used, having $\mathrm{Cu}$ line. Standard runs using forty $\mathrm{kV}$ voltage, a twenty five 
$\mathrm{mA}$ current and a scanning rate of $1^{\circ}$ min- 1 over $2 \theta$ vary of $10-70^{\circ}$ were used

\subsubsection{Vesicles shape}

Surface morphology of drug Nanocrystal formulation was examined at $\mathrm{pH}$ (7.4) and interpreted using scanning electron microscope (Jeol JSM-840A Japan). The sample to be analyzed were placed on metal mount (Aluminum) and thus were cleaned, and were coated with nine nm of gold / palladium using a unit which is sputter-coated were recorded and analyzed using SEM.

\subsubsection{In vitro drug release studies}

RVT Nanocrystal's release level was calculated using the (Basket type) type-I(United State Pharmacopeia) dissolution testing apparatus. The in vitro release profile (dissolution test) was conducted with a pH (1.2) buffer of $900 \mathrm{ml}$ at $37 \pm 0.5^{\circ} \mathrm{C}$ and $50 \mathrm{rpm}$. All formulations of drugs and pure drugs were individually filled in capsules and were subjected to dissolution study. A sample $(5 \mathrm{ml})$ of the solution was collected from the dissolution system for 1 hour after every 15 minutes interval having $0.1 \mathrm{~N} \mathrm{HCL}$ as dissolution media containing sodium lauryl sulphate in concentration of $0.25 \% \mathrm{w} / \mathrm{v}$, which was further followed by 30 minutes interval for the next 5 hours. Absorbance of each solution was recorded against buffer solutions at $244 \mathrm{~nm}$ using UV Spectrophotometer (Shimadzu's UV-1900i) and percentage drug release was calculated for each formulation.

\subsection{RESULT AND DISCUSSION}

\subsection{Analytical method for the estimation of RVT using UV spectrophotometer}

The standard calibration plots of the drug were prepared in $0.1 \mathrm{~N}$ hydrochloric acid solutions and phosphate buffer solution $\mathrm{pH}$ 6.8. The solutions of lornoxicam were scanned by the UV spectrophotometer at the wavelength range of $800-400 \mathrm{~nm}$. The $\lambda$ max of drug solution is $244 \mathrm{~nm}$ in $0.1 \mathrm{~N}$ hydrochloric acid solutions and phosphate buffer solution $\mathrm{pH}(6.8, \mathrm{pH} 7.4)$

\subsection{Solubility Study}

RVT saturation solubility was performed for estimation in different buffer solutions to select appropriate dissolution medium for in vitro release studies. The saturation solubility of RVT in different media is shown in Table 3.

Assessment of saturation solubility in distilled water, $0.1 \mathrm{~N}$ $\mathrm{HCl}$ (pH 1.2), phosphate buffer $\mathrm{pH}$ (6.8) \& phosphate buffer $\mathrm{pH}$ (7.4) was carried out. An excess amount of rosuvastatin calcium (pure drug) and Nanocrystal formulation of drug were separately dispersed into $10 \mathrm{ml}$ of medium in a conical flask. The mixture was stirred in orbital shaker incubator for $24 \mathrm{hrs}$ at a constant temperature of $37.0 \pm 0.5{ }^{\circ} \mathrm{C}$. The sample was analyzed (after desired dilution) using UV spectrophotometer at $244 \mathrm{~nm}$. The solubility data obtained for each formulation were calculated as an average of three measurements.

In this study, kinetics of saturation solubility of rosuvastatin calcium (pure drug) and Nanocrystal formulation of drug were studied in the different dissolution media. The results of solubility study (Table 2) indicated increase in saturation solubility of Nanocrystal formulation of drug in the selected dissolution media. The Nanocrystal formulation of rosuvastatin calcium when compared with pure drug exhibited approximately three times and eight times increase in saturation solubility in distilled water and $0.1 \mathrm{~N}$ $\mathrm{HCl}$ respectively. This could be attributed to increase in surface area and the presence of pores/channels (as evident from SEM images) in the Nanocrystal formulation of rosuvastatin calcium.

Table 2: Saturation solubility of rosuvastatin calcium and Nanocrystal formulation of rosuvastatin calcium

\begin{tabular}{|l|l|l|}
\hline Media & RSV $(\mathrm{mg} / \mathrm{ml})$ & PSNC-3(mg/ml) \\
\hline Distilled Water & $3.77 \pm 0.85$ & $9.51 \pm 1.67$ \\
\hline $0.1 \mathrm{~N} \mathrm{HCl}$ & $0.63 \pm 0.21$ & $4.09 \pm 1.09$ \\
\hline $\begin{array}{l}\text { Phosphate buffer } \\
(6.8)\end{array}$ & $1.08108 \pm 0.005$ & $7.52 \pm 1.77$ \\
\hline $\begin{array}{l}\text { Phosphate buffer } \\
\text { pH(7.4) }\end{array}$ & $2.19107 \pm 0.0082$ & $8.71 \pm 1.57$ \\
\hline
\end{tabular}

\subsection{Particle Size Distribution}

Particle size, polydispersity index (PDI) and zeta potential of rosuvastatin (RVT) and RVT formulated with different stabilizers as shown in (Table-3) were determined using Delsa Nano ${ }^{\mathrm{TM}}$ (Beckman Coulter, Germany) equipment.

The particle size of RVT and PSNC-3 was found to be 1975.3 $\mathrm{nm}$ and $790.1 \mathrm{~nm}$ respectively as shown in figure-1. The nanocrystals were characterized with respect to practical yield and particle size. The particle size and the width of the PSD are important characterization parameters as they govern in-vitro parameters such as saturation solubility, physical stability, drug dissolution and and in-vivo performance of nanoparticles. The PSD of PSNC3nanocrystals are shown in Table 2 .. The results from clearly suggest that as drug: polymer ratio increased significant reduction in particle size of the drug could be correlated with the enhanced solubility/dissolution characteristics of nanocrystals of the drug. Nanoformulations offer the advantage of enhanced bioavailability at lower dosages, thus reducing side effects and hepatic load. Nanosized drug crystals provide a greater surface area for dissolution and increase the saturation solubility, ultimately increasing dissolution, which leads to enhanced absorption in vivo. Commercial concerns relate to safety, because this affects regulatory approvals. The term 'nano' by itself is too wide and in the context of nanoformulations, there has to be correctly defined 'range' which is considered 'safe' for approval, from a regulatory perspective. Transport of nanocarriers into lymphatic vessels is dependent on particle size. Nano carrier size lies between $200-800 \mathrm{~nm}$ to avoid uptake by the reticulo-endothelial system. Oussoren et al[23] reported that a particle size of $10-100 \mathrm{~nm}$ is optimal for lymphatic uptake. The mean particle for different formulations available lies between $300-800 \mathrm{~nm}$. As physicochemical properties of drugs vary, therefore there is a need for the size to be optimized for different API'S. In case of present formulation, the particle size is about $700 \mathrm{~nm}$ i.e. well within the safe limit.

The PDI and zeta potential results indicated that lyophilized nanocrystal formulation was physically stable. 
Table 3: Particle size, polydispersity index (PDI) and zeta potential of rosuvastatin (RSV) and various Nanocrystal formulations

\begin{tabular}{|l|l|l|l|l|l|}
\hline $\begin{array}{l}\text { Formulation } \\
\text { code }\end{array}$ & Stabilizer & $\begin{array}{l}\text { Drug:Stabilizer } \\
\text { ratio }\end{array}$ & $\begin{array}{l}\text { Particle } \\
\text { size(nm) }\end{array}$ & $\begin{array}{c}\text { Zeta Potential } \\
(\mathbf{m V})\end{array}$ & PDI \\
\hline Pure Drug & & & 1975.3 & -14.61 & 0.790 \\
\hline PNC-1 & Polaxmer 188 & $1: 1$ & 1073.2 & -18.56 & 0.856 \\
\hline PNC-2 & & $1: 2$ & 1123.1 & -17.75 & 0.968 \\
\hline PNC-3 & & $1: 3$ & 934 & -15.61 & 0.798 \\
\hline SNC-1 & SLS & $1: 1$ & 1235 & -14.63 & 0.545 \\
\hline SNC-2 & & $1: 2$ & 1345 & -19.61 & 0.654 \\
\hline SNC-3 & & $1: 3$ & 956 & -9.69 & 0.786 \\
\hline PSNC-1 & SLS: Polaxmer 188 & $1: 1$ & 956 & -14.61 & 0.445 \\
\hline PSNC-2 & & $1: 2$ & 863.2 & -10.26 & 0.346 \\
\hline PSNC-3 & & $1: 3$ & 790.2 & -14.61 & 675 \\
\hline
\end{tabular}

PNC= Polaxmer nanocrystals , SNC= Sodium lauryl sulphate nanocrystals, PSNC= Polaxmer Sodium lauryl sulphate nanocrystals

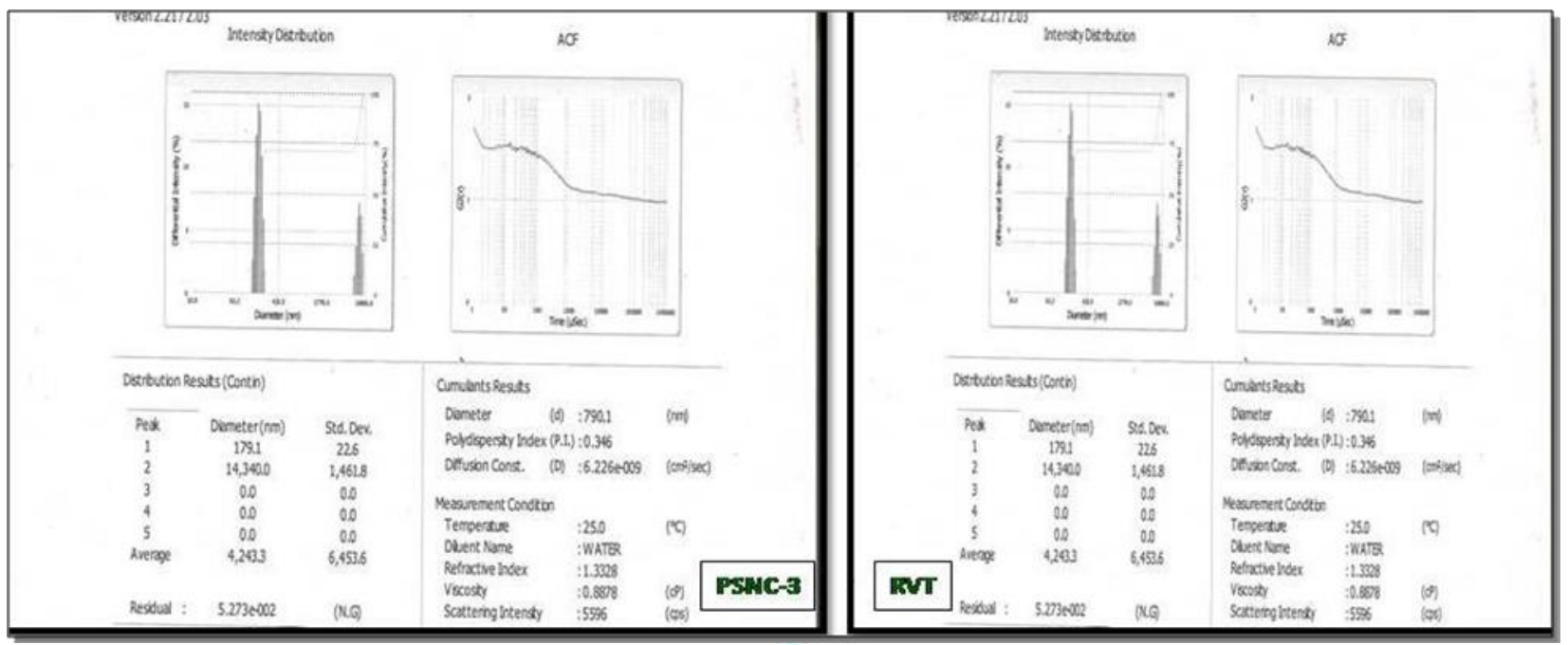

Figure 1 Particle size distribution of pure drug and Drug nanocrystals

\subsection{Differential scanning calorimetry (DSC):}

It is well documented that DSC i,e Differential Scanning Calorimetry, is explored to analyze and forecast changes in drug's crystallinity. Polymorphism may interpret as substance's ability as well tendency to exist in one or more crystalline forms. Alterations in melting point, variation in bioavailability and drug release are expected due to change in drugs polymorphic nature. Due to its phase transition, a characteristic large and sharp peak was observed at $175.19^{\circ}$ for Rosuvastatin Calcium At $171.44^{\circ}$ and $180.59^{\circ}$ respectively, the start and end of the RVT phase transition array are observed. DSC formulation thermogram, PSNC-1 showed an endothermic peak at $173.92{ }^{\circ} \mathrm{C}$ which may be lead to interpret as a slight change in crystalline nature. Formulations [A] (PSNC-2, [B] (PSNC-3) having composition as shown in (Table 3), DSC thermograms displayed small but sharp characteristic endothermic peak at $174.87^{\circ}$ and 174.57 respectively. Based on results obtained and interpretation of DSC thermogram of formulation which may be considered for further studies displayed characteristic endothermic peaks corresponding to those of the pure drug, and one or more new peaks or disappearances corresponding to those of the pure drug do not occur.

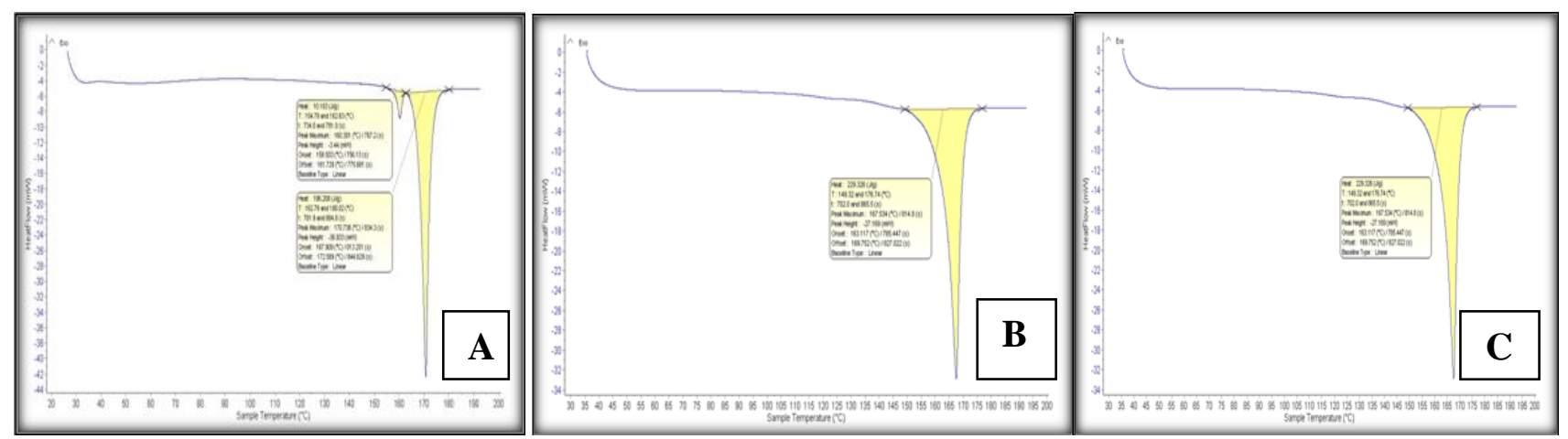

Fig:2 DSC Patterns of pure RVT (A) ,(B) PSNC-2, (B) PSNC-3. 


\subsection{XRD Analysis}

XRD measurement was performed by X-ray diffractometer (Bruker, Germany) over the range of angle 2-theta of 5 to $40^{\circ}$ with a step width of $0.04^{\circ}$ and a count time of $4 \mathrm{~s}$. An amorphous form of a compound intended for pharmaceutical use may give rise to manufacturing problems and there is need to identify crystalline forms of these compounds which may have different physical characteristics compared to amorphous form. Crystalline forms of such compounds may also possess improved physical, chemical and pharmacological characters such as improved solubility/dissolution, enhanced bioavailability etc. XRD spectra of PSNC-3 clearly indicate the presence of additional sharp peaks. Moreover, splitting of peak (specifically near to $2 \theta$ value of 20 ) could be seen in PSNC-3 when compared with RSV. It point out towards the increase in crystalline behavior of PSNC-3 as compared to RVT.

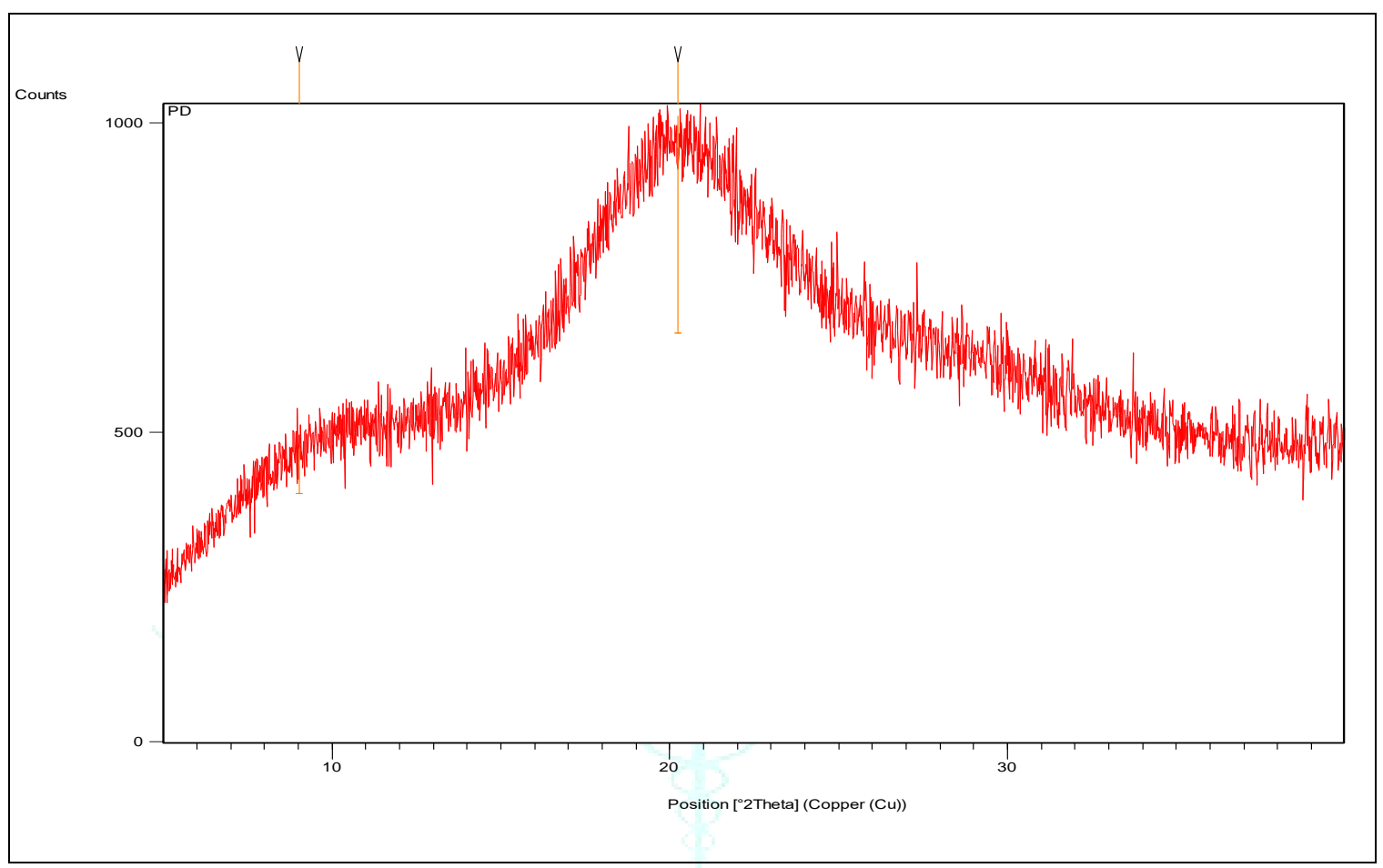

(A)

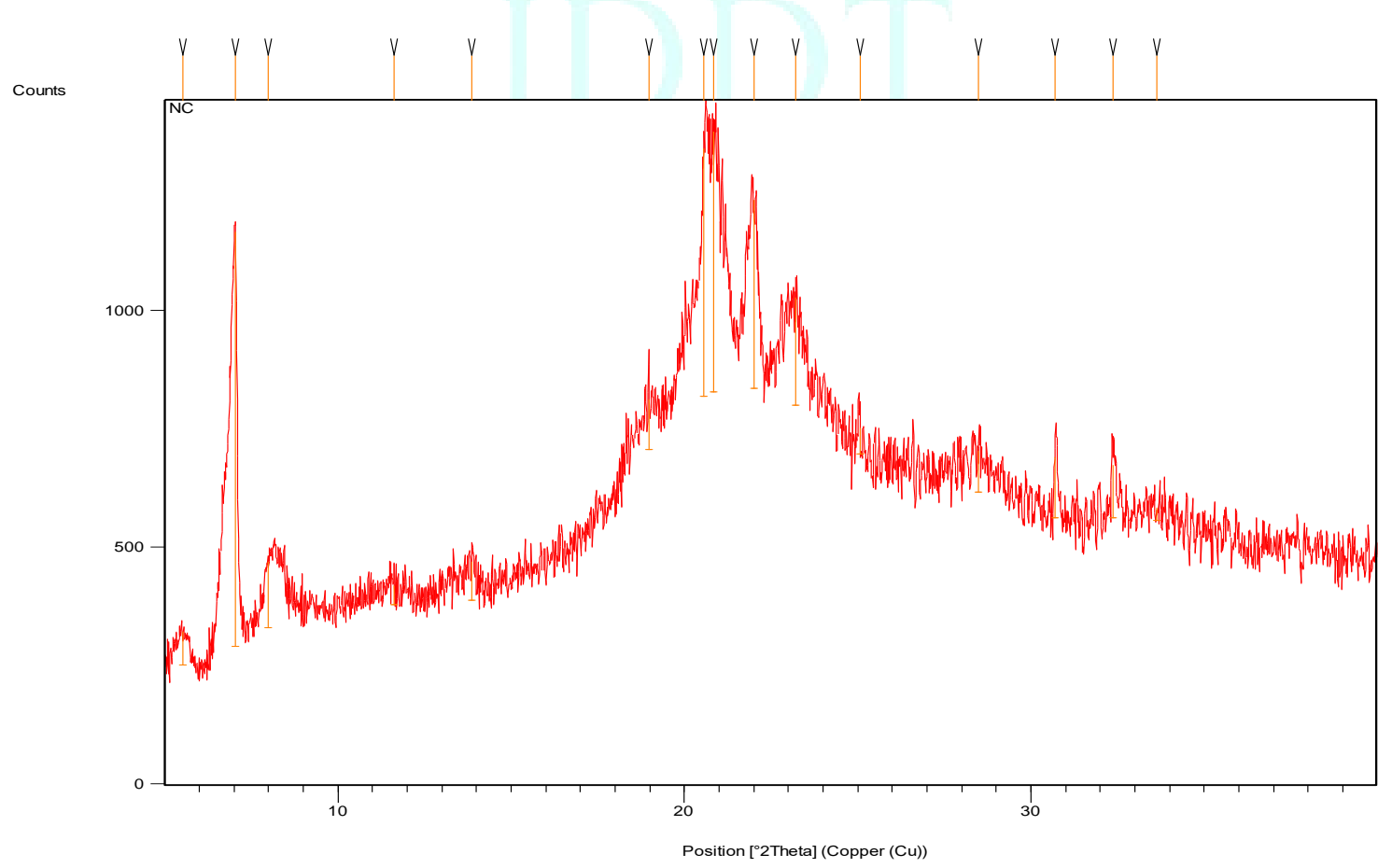

(B)

Figure 3: XRD spectra of (A) rosuvastatin calcium (B) PSNC-3 
Table 4: XRD analysis parameters $(2 \theta, \mathrm{d}$ spacing, relative intensity) of rosuvastatin calcium (RSV) and Nanocrystal formulation of rosuvastatin calcium (PSNC-3).

\begin{tabular}{|c|c|c|c|}
\hline \multicolumn{3}{|c|}{ RSV } \\
\hline S. No. & $2 \theta$ & d spacing & Relative Intensity \\
\hline 1. & 9.03 & 9.78 & 21.14 \\
\hline 2. & 20.23 & 4.38 & 100 \\
\hline \multicolumn{3}{|c|}{ PSNC-3 } \\
\hline 1. & 5.53 & 15.95 & 8.05 \\
\hline 2. & 7.04 & 12.53 & 100 \\
\hline 3. & 7.99 & 11.05 & 15.97 \\
\hline 4. & 20.56 & 4.31 & 59.96 \\
\hline 5. & 20.85 & 4.25 & 62.89 \\
\hline 6. & 22.01 & 4.03 & 45.55 \\
\hline 7. & 23.21 & 3082 & 25.99 \\
\hline 8. & 30.69 & 2.91 & 14.68 \\
\hline 9. & 32.35 & 2.76 & 12.64 \\
\hline
\end{tabular}

\subsection{Scanning Electron Microscopy (SEM Analysis)}

The morphological characteristics of rosuvastatin calcium and Nanocrystal formulation of rosuvastatin calcium were examined using scanning electron microscope.
SEM images of pure RVT indicates compact and discrete surface. However, SEM images of PSNC-3 shows the presence of channels and pores. Increased surface area could also be seen in the images.
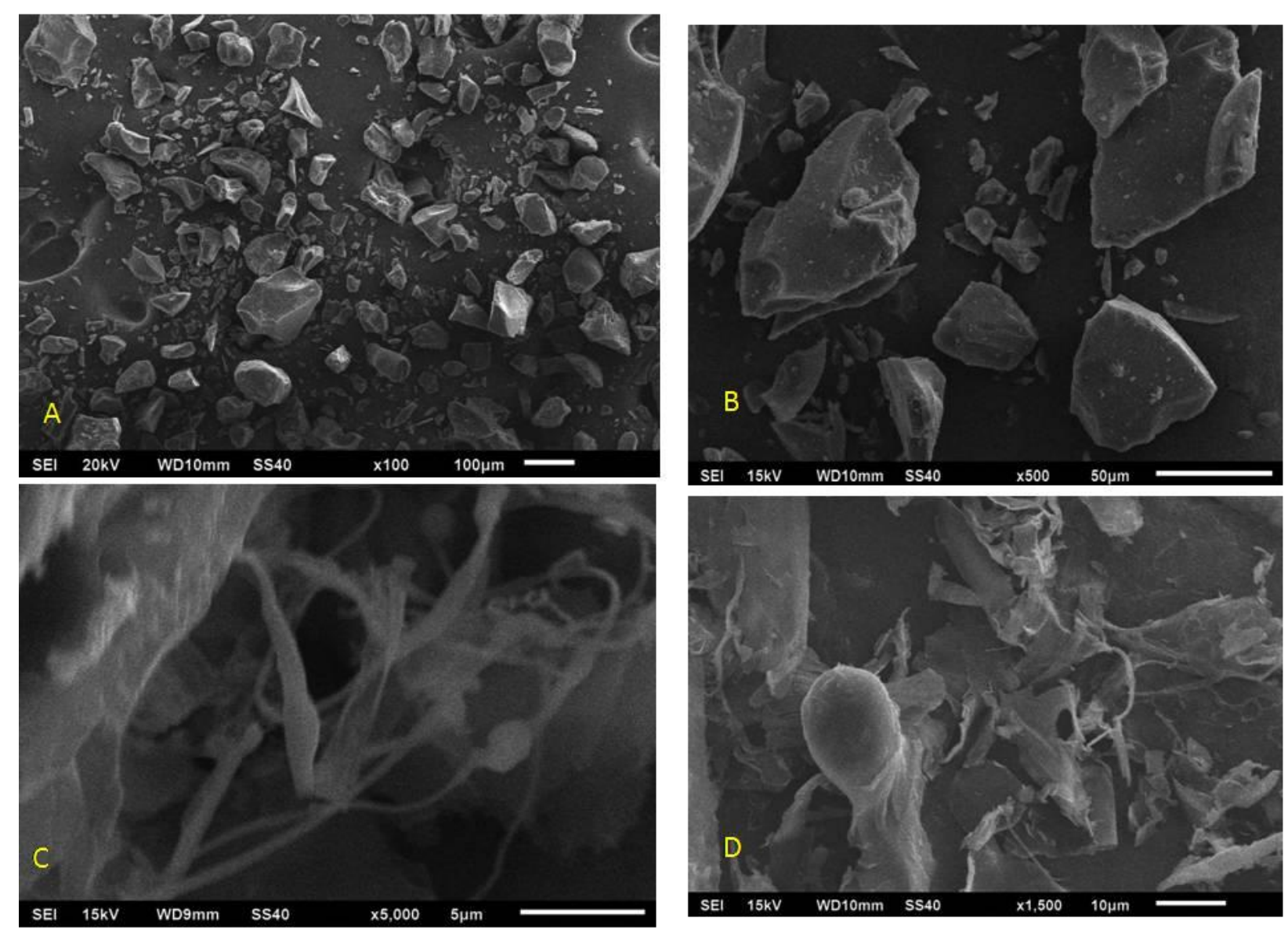

Figure 4: Scanning electron microscopy images of pure drug (A\&B) and PSNC-3 (C\&D) 


\subsection{In vitro drug release studies}

The in vitro dissolution of rosuvastatin calcium and Nanocrystal formulation of rosuvastatin calcium from tablet and capsule formulation was determined using USP Apparatus 2 dissolution tester (Labindia, Mumbai, India). The dissolution test was conducted in $0.1 \mathrm{~N} \mathrm{HCl} 900 \mathrm{ml}$. The speed of the paddle was $50 \mathrm{rpm}$. Holding the temperature at $37 \pm 0.5^{\circ} \mathrm{C}$ also at the predetermined time points, five milliliters of dissolution medium is obtained from the vessel. The collected sample was immediately filtered through a membrane filter of $0.2 \mu \mathrm{m}$ and fresh dissolution media were replenished into the vessel immediately. The samples were using UV spectrophotometer at $244 \mathrm{~nm}$. For each sample, three replicate dissolution tests were performed.
The drug release (after 15 minutes) from tablet formulation of RVT and PSNC-3 (optimized formulation) was nearly $12 \%$ and $32 \%$ respectively. After 60 minutes the drug release was found to be $41 \%$ and $83 \%$ from tablet formulation of RSV and PSNC-3 respectively. In case of capsule formulation of RSV and PSNC-3, the drug release was found to be approximately $10 \%$ and $40 \%$ respectively after 15 minutes. After 60 minutes the drug release was found to be $41 \%$ and $83 \%$ from capsule formulation of RSV and PSNC-3 respectively. The results suggested that the PSNC-3 had a significant effect on the drug solubility, dissolution rate, and dissolution behavior.

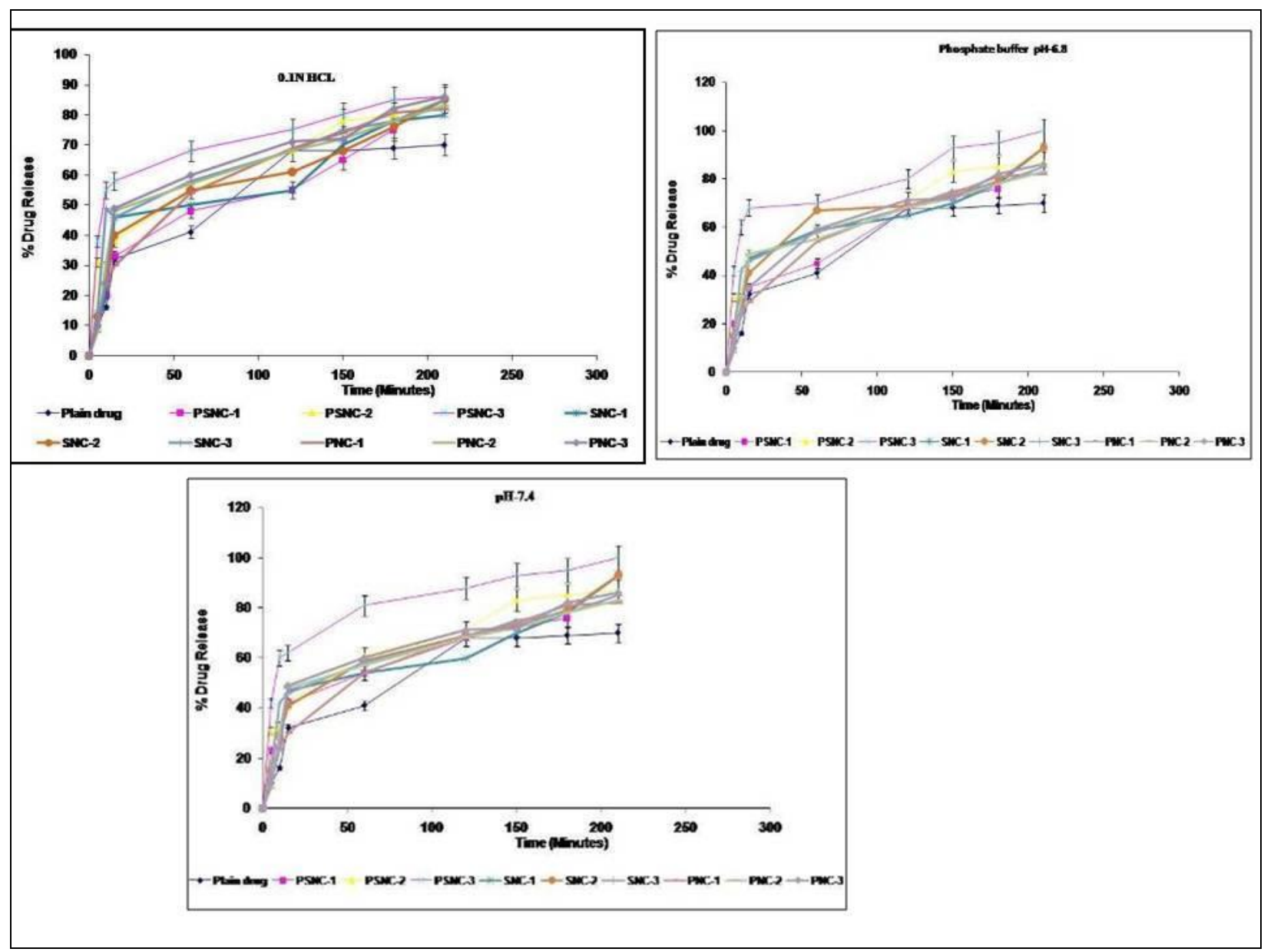

Figure 5:- In vitro dissolution profile of rosuvastatin calcium and Nanocrystal formulation with different stabilizers in different media

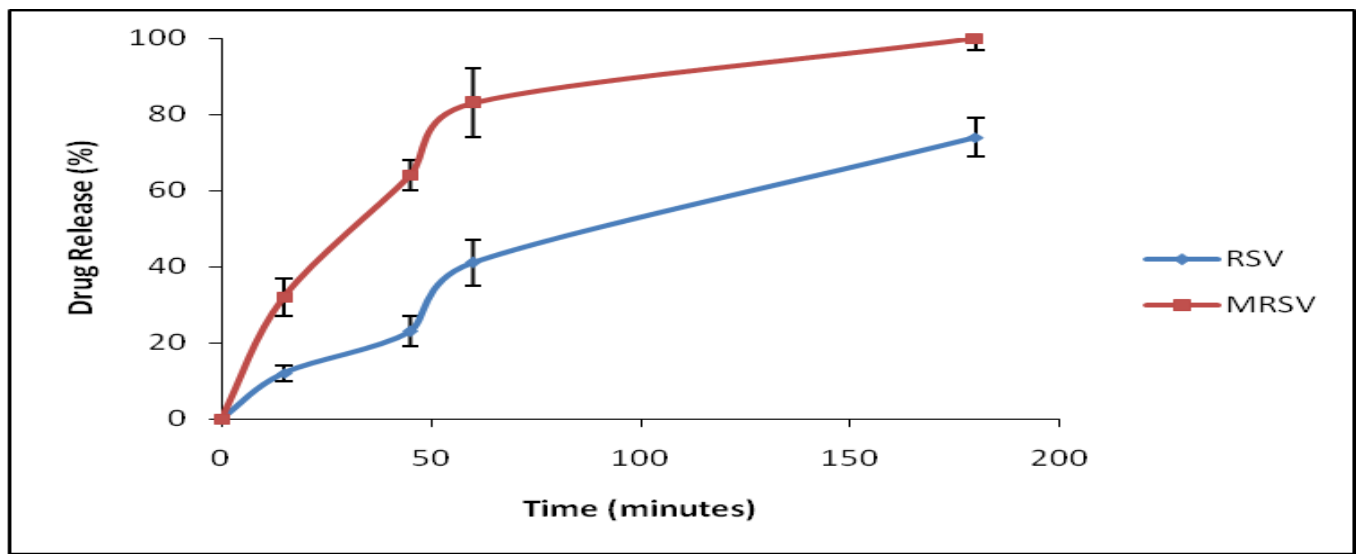

Figure 5: In vitro dissolution profile of rosuvastatin calcium and PSNC-3 tablets 


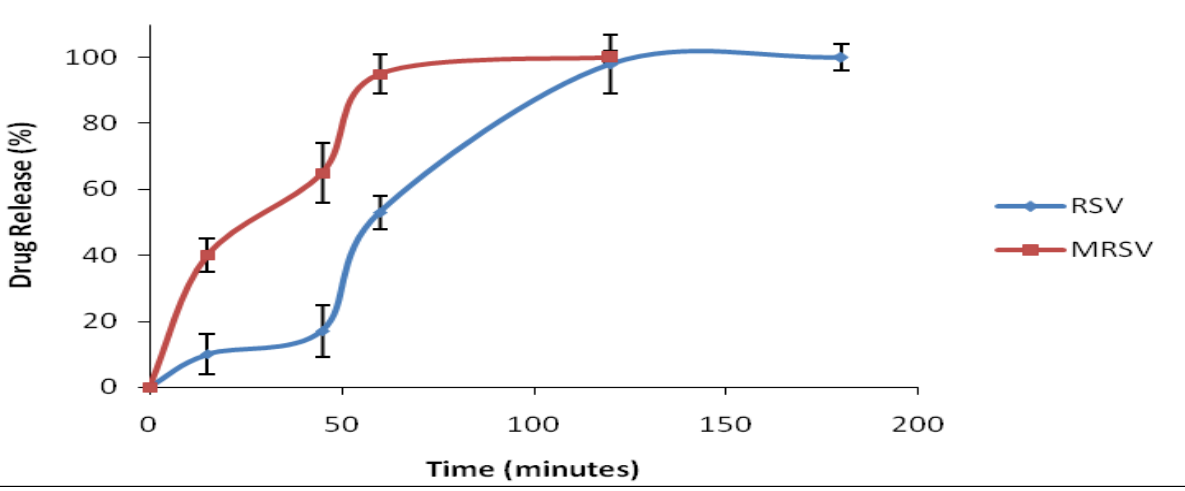

Figure 6: In vitro dissolution profile of rosuvastatin calcium and PSNC-3 capsules

\subsection{CONCLUSION}

Rosuvastatin calcium is an Anti-hyperliperdemic drug of the statin class with analgesic belongs to BCS Class IV Therefore; an attempt has been made to improve solubility by converting pure drug of RVT $m$ which is in micronized form to nanosized form. Nanocrystals of RVT were developed with different ratios of SLS, polaxmer 188, by antisolvent precipitation method. The FTIR spectroscopy was used to confirm compatibility and to rule out any possible interactions between drug and polymers. Nine nanocrystal formulations (SNC-1,SNC-2) SNC-3,PNC-1, PNC-2, PNC3,SPNC-1,SPNC-2,SPNC,3) consisting pure drug of RVT (micronized form) with SLS, polaxmer 188 used as stabilizers in the ratios of $1: 1,1: 2$, and $1: 3$, respectively,

All formulations carried using acetonitrile and double distilled water as antisolvent system. The prepared nanocrystals were evaluated for their physicochemical characteristics such as physical appearance, FTIR, DSC, SEM, XRD, solubility studies, PSD, zeta potential, and in vitro drug release studies. In vitro drug release from nanocrystals was carried out in different buffers, and the data obtained were fit into different equations and kinetic models to explain release kinetics. Rosuvastatin with SLS, polaxmer 188 in 1:3 ratio formulations in $0.1 \mathrm{~N}$ Hcl \& $7.4 \mathrm{pH}$ phosphate buffer and showed better solubility and emerged to be ideal formulation for Rosuvastatin calcium nanocrystals. Hence, it can be concluded that optimized nanocrystals formulation of RVT improved the solubility and dissolution of Rosuvastain calcium as compared to pure drug.

\section{REFERENCES}

1. Huttunen, K.M., Raunio, H., Rautio, J., Prodrugs - from serendipity to rational design. Pharmacol. Rev. 2011; 63:750-771

2. Serajuddin, A.T., Salt formation to improve drug solubility. Adv. Drug Deliv. Rev. 2007; 59:603-616

3. Seedher, N., Kanojia, M., Co-solvent solubilization of some poorlysoluble antidiabetic drugs. Pharm. Dev. Technol. 2009; 14:185192

4. Bilensoy, E., Hincal, A.T., Revent advances and future directions in amphiphilic cyclodextrin nanoparticles. Expert Opin. Drug Deliv. 2009; 6:1161-1173

5. Thakuria, R., Delori, A., Jones, W., Lipert, M.P., Roy, L., RodriguezHornedo, N., Pharmaceutical cocrystals and poorly soluble drugs. Int. J. Pharm. 2013; 453:101-125

6. Babu, N.J., Nangia, A., Solubility advantage of amorphous drugs and pharmaceutical cocrystals. Cryst. Growth Des. 2011; 11:2662-2679.

7. Tuomela, A., Laaksonen, T., Laru, J., Antikainen, O., Kiesvaara, J., Ilkka, J., Oksala, O., Rönkkö, S., Järvinen, K., Hirvonen, J., Peltonen, L., Solid formulations by a nanocrystal approach: critical process parameters regarding scale-ability of nanocrystals for tableting applications. Int. J. Pharm. 2015; 485:77-86.

8. Thi, T.D., Van Speybroeck, M., Barillaro, V., Martens, J., Annaert, P., Augustijns, P., Van Humbeeck, J., Vermant, J., Van den Mooter, G., Formulate-ability of ten compounds with different physicochemical profiles in SMEDDS. Eur. J. Pharm. Sci. 2009; 38:479-488.

9. Shakeel, F., Faisal, M.S., Nanoemulsion: a promising tool for solubility and dissolution enhancement of celecoxib. Pharm. Dev. Technol. 2010; 15:53-56.

10. Porter, C.J.H., Trevaskis, N.L., Charman, W.N., Lipids and lipidbased formulations: optimizing the oral delivery of lipophilic drugs. Nat. Rev. 2007; 6:231-248.

11. Shakeel, F., Faisal, M.S., Nanoemulsion: a promising tool for solubility and dissolution enhancement of celecoxib. Pharm. Dev. Technol. 2010; 15:53-56.

12. Chen, H., Khemtong, C., Yang, X., Chang, X., Gao, J., Nanonization stragegies for poorly water-soluble drugs. Drug Discov. Today 2011; 16:354-360

13. Brough, C., Williams III, R.O., Amorphous solid dispersions and nano-crystaltechnologies for poorly water-soluble drug delivery. Int. J. Pharm. 2013; 453:157-166

14. Loftsson, T., Brewster, M.E., Pharmaceutical applications of cyclodextrins: basic science and product development. J. Pharm. Pharmacol. 2010; 62:1607-1621

15. Brouwers, J., Brewster, M.E., Augustijns, P., Supersaturating drug delivery systems: the answer to solubility-limited oral bioavailability? J. Pharm. Sci. 2009; 98:2549-2572

16 Liu, P., Rong, X., Laru, J., van Veen, B., Kiesvaara, J., Hirvonen, J., Laaksonen, T., Peltonen, L., Nanosuspensions of poorly soluble drugs: preparation and development by wet milling. Int. J. Pharm. 2011; 411:215-222.

17. Borchard, G., 2015. Drug nanocrystals. In: In: Crommelin, D., de Vlieger, J. (Eds.), Non-Biological Complex Drugs. AAPS Advances in the Pharmaceutical Sciences Series, vol. 20. Springer, Cham, pp. 171-189

18 Kesisoglou, F., Panmai, S., Wu, Y., Nanosizing - oral formulation development and biopharmaceutical evaluation. Adv. Drug Deliv. Rev. 2007; 59:631-644

19. Gao, L., Liu, G.Y., Ma, J.L., Wang, X.Q., Zhou, L., Li, X., Drug nanocrystals: in vivo performances. J. Control. Release 2012; 160:418-430.

$20 \mathrm{Lu}, \mathrm{Y} ., \mathrm{Li}, \mathrm{Y} ., \mathrm{Wu}, \mathrm{W}$., Injected nanocrystals for targeted drug delivery. Acta Pharm. Sin. B 2016; 6:106-113.

21 R.R. Sevda, A.S. Ravetkar, P.J. Shirote, UV spectrophotometric estimation of Rosuvastatin calcium and fenofibrate in bulk drug and dosage form using simul-taneous equation method, Int. J. Chem. Tech. Res. 2011; 3:629-635.

22. Fu, Q., Sun, J., Zhang, D., Li, M., Wang, Y., Ling, G., Liu, X., Sun, Y., Sui, X., Luo, C., Sun, L., Han, X., Lian, H., Zhu, M., Wang, S., He, Z., Nimodipine nanocrystals for oral bioavailability improvement: preparation, characterization and pharmacokinetic studies. Colloids Surf. B Biointerfaces 2013; 109:161-166.

23 Oussoren C1, Storm G. Liposomes to target the lymphatics by subcutaneous administration. Adv Drug Deliv Rev. 2001; 50(12):143-56 